\title{
A KLYSTRON-MODULATOR INTERLOCK AND FAULT ACQUISITION SYSTEM USING G-64, VME, AND MIL-1553-B STANDARDS
}

\author{
A. Campbell*, M. Grippeling**, \\ G. McMonagle, P. Pearce, G. Rentier
}

\begin{abstract}
A new microprocessor controlled protection system has been integrated with the control and data acquisition scheme of the $35 \mathrm{MW}, 100 \mathrm{~Hz}$ klystron-modulators for the pre-injector (LPI) of the Large Electron Positron (LEP) at the European Laboratory for Particle Physics (CERN), near Geneva. This equipment replaces the original relay logic hardware and is designed to improve the reliability and functionality of the modulators without compromising any safety aspects. Further, it acts as a base for the recently implemented data acquisition scheme which collects and stores modulator fault information.

In this paper we describe the G-64 hardware design and the firmware program structure that assures continuous, and fast surveillance of all interlock signals. This firmware program also prepares and stores any fault data acquisition by a real time task, written in $C$ language, and operating within a VME hardware environment. A collector program will access this data and transfer it to the main ORACLE data base for use by high level maintenance and operator programs. The communication interface used between the G-64 and VME systems is the MIL-1553-B standard.
\end{abstract}

* Strathclyde University, United Kingdom, **Alkmaar Technical University, The Netherlands

This paper is to be presented at the 1994 Twenty first Power Modulator Symposium, COSTA MESA, CA., USA 


\section{A KLYSTRON-MODULATOR INTERLOCK AND FAULT ACQUISTTION SYSTEM USING G-64, VME, AND MIL-1553-B STANDARDS}

A. Campbell, Strathclyde University, UK, M. Grippeling, Alkmaar Technical University, The Netherlands, G. McMonagle, P. Pearce, G. Rentier, CERN, Geneva, Switzerland.

\section{Introduction}

The IPI pre-injector is the first machine in a series chain of four accelerators that provides e-e beams for the Large Electron Positron Collider(LEP) at the European Laboratory for Particle Physics (CERN) near Geneva. The klystron-modulators in the LPI preinjector operate at $100 \mathrm{H}$ rate injector operate at $100 \mathrm{~Hz}$ rate and each provides up to IW of peak $R$ power for the acceleration of the electron beams. Over a period of time, and with continuous system usage, components, active elements and sub-systems in the klystron-modulators start to age and have caused the overall rellabllity and avallability of the process to degrade. The reliability of the total process and the availability of each accelerator in this series production chain is dependent on the rellability of every individual cubsystem in the ref the four subsystem in each of the lour accelerators. The overall process reliability $R_{s}$ is given by an terative product rule that uses the rellability of all elements $R_{1}$ in this series chain, regardless of the particular failure distributions of components used.

The reliability iterative product rule is given by

$$
R_{s}=\prod_{i=1}^{n} \cdot R_{i}
$$

The greater the number of series elements in the chain, the lower will be the process reliability. More imoortantly, the overall reliability of the total system becomes lower than that of the weakest system system becomes
or sub-system.

The interlock relay protection sub-systems used in each of the LPI kIystron-modulators, and part of the chain, were found to be increasingly contributing to the downtime and unreliability of the lepton production process. This paper describes the reliability problem and the design of a new replacement interlock protection systern. The new design uses a micro-processor that has greatly improved modulator reliablitty and has added extra improved modulator reliability and has

\section{Interlock syatem problems}

Each modulator interlock system is designed to protect the hardware from excessive damage if a fault occurs, and so must have an inherent high level of reliability and so must have an inherent high level of reliability to function correctly. Up to 120 input signals, coming from semi-static and real time operational subcontinuously processed. In the original scheme the input signals operate 120 separate d.c. relays in each interlock unit. The output relay contacts are organised into four separate Interlock chains, corresponding to the four output control levels loff, Heater, Standby, Pulsing). A d.c current of about $20 \mathrm{~mA}$ llows through every contact in each of the series chains of contacts, enabling the four output protection conditions.

During normal production operation the modulator system works continuously for many months at a time. syctem works continuously for many months at a time. occurred, mainly due to contact oxidisation since the occurred, mainly due to contact oxidisation since the relays stay in an activated closed position. This intermittent oxidisation build-up increased the system
downtime in a randor fashion. In addition, because most individual interlocks are not memorised the fault finding and separation of true external faults from internal contact problens was difficult.

To overcome these difficulties a more reliable system has been designed using comercial solid state G64 micro-processor and opto-coupled interface board micro-processor and opto-coupled intertace board technology to replace the relay logic system. The new scheme, apart from improving modulator reliability can store up to elght complete fault sequences in local each sequence the first eight faulty interlocks are memorised together with a date and time tag to give maximum fault finding information.

\section{Hicro-processor interlock aystem}

The design requirements for the new interlock protection system were:

- Complete electrical compatibility with old system

- A fall-safe design approach

- High reliabillty

- An ordered fault memorisation

- Interfacing to control system and Oracle database

- Conforming to CER personnel security requirements

\section{Hardware design}

A stand-alone protection system (1) has been developed that uses the MC6809, 8 bit micro-processor within a G-64 data bus crate. This system handles the 120 input signals via purpose-designed 32-way input buffer cards. Each input interlock signal generates three output signals. These are used for the following purposes:

1) A direct interlock connection to the 32 way input card for modulator protection

2) An opto-isolated output for the existing CakAC data acquisition system

3) A TTL level output for on-board testing purposes

The new micro-processor interlock protection system is shown in Figure 1.

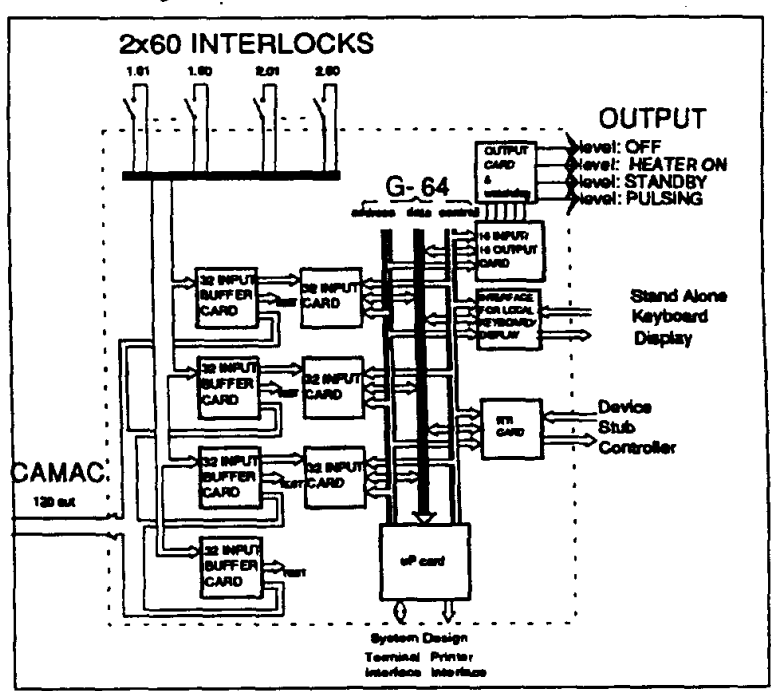

Figure 1 Micro-processor controlled interlock herdware scheme 
The 32 way input card handles the interlocks in groups of eight and any one of these groups can generate a hardware interrupt for the processoz.

The failed interlocks are then collected in groups of eight by the program and stored in a local memory. Any interrupt is processed immediately by an interrupt service routine (ISR), which changes the output status as required.

The MC6809 micro-processor has sixteen address lines so that a total of 64kbytes of memory can be addressed. The actual program is stored in EPROM which holds a maximum of 28xbytes. The same EPROM also stores the interrupt and start vector addresses. The 16 way output board connects the G-64 system with the 16 way output board connects the G-64 system with the modulator via opto-coupled links and a buffer card. There are four output levels that react directly with the modulators power circuits and control its status. monitors the functioning of the processor via a status line that is toggled by the software. If this line stops toggling, the program has stopped running and the protection system is no longer working. Under these conditions the modulator is immediately put to the OFF state. The machine access control and HV protection doors also provide interlock contacts that protection doors also provide interlock contacts that
are connected in series with the output control levels to give additional personnel security

ERROM software development

The main interlock management program is a loop that includes the accuisition and treatment of the 120 includes the acculsition and treatment of the 120 diagram of this program is given in Figure 2. The diagram of this program is given in Figure 2. The program 'display' variable is used to make sure that the display screen infomation is written without occurs during this operation. The initialisation, start-up and interrupt routines have been written in assembler micro-code.

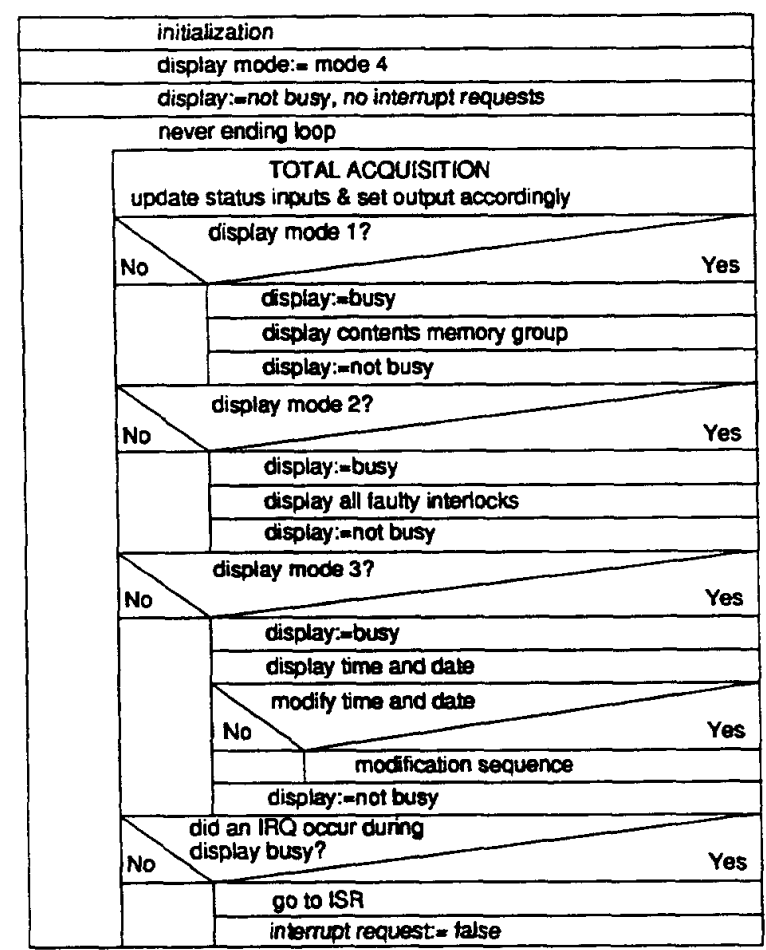

rigure 2 kain program flow diagram
There are two sources of system interrupt that can occur. One that is generated by a faulty interlock and a second by a key on the units display panel. However, the interlock generated interrupts have priority over any display requests. The response time to an interlock generated interrupt is shown in Figure 3.

The delay between the switching of the input interlock and the response of the NOR gate is caused by the opto-coupler. The overall response time between the change of input and the $\overline{\mathrm{RQQA}}$ line on the G-64 bus is about 14 ps.

The four channels represent the following signals:

CH1 (A): Input interlock signal.

CH2 (B): output of a group of eight interlocks CH3 (C): Interrupt flag

CH4 (D): 664 interrupt request line (IRQA).

\section{0-Apr-93}

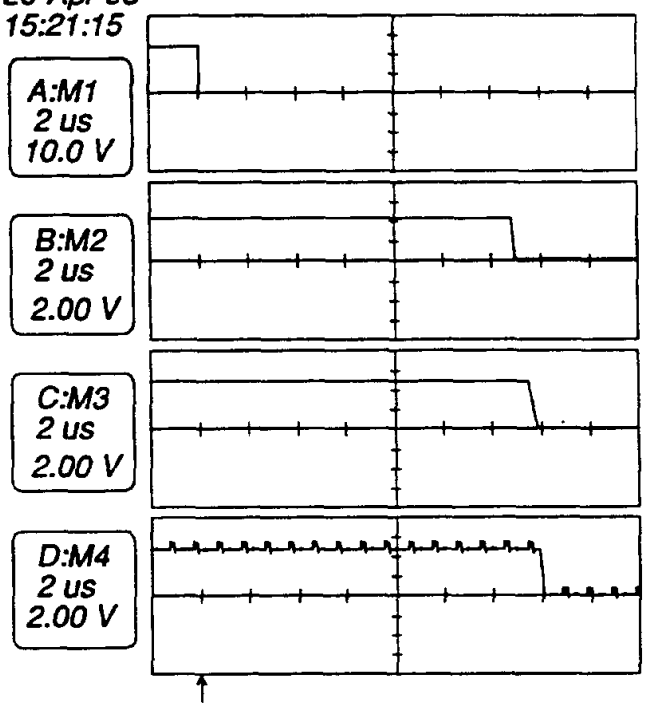

Figure 3 Interlock noquisition response time.

When a fault occurs the interrupt service routine is executed and any subsequent new interrupts are disabled wilst the input group that caused the first interrupt is traced by means of a polling routine. The output status of the protection system is switched to the assigned operating level and so protecting the modulator. Finaliy the interfupt flag is cleared and the faulty interlock is stored along with the date and tine tags.

The display screen on each interlock unit allows the following display modes to be selected :

- Mode 1 Checks system memory and is automatically activated when pulsing.

- Mode 2 Displays current status of interlocks.

- Mode 3 Changes the date and time.

- Mode 4 Displays information and error messages.

Each of the four display modes have their own software driver module containing special functions and procedures. 


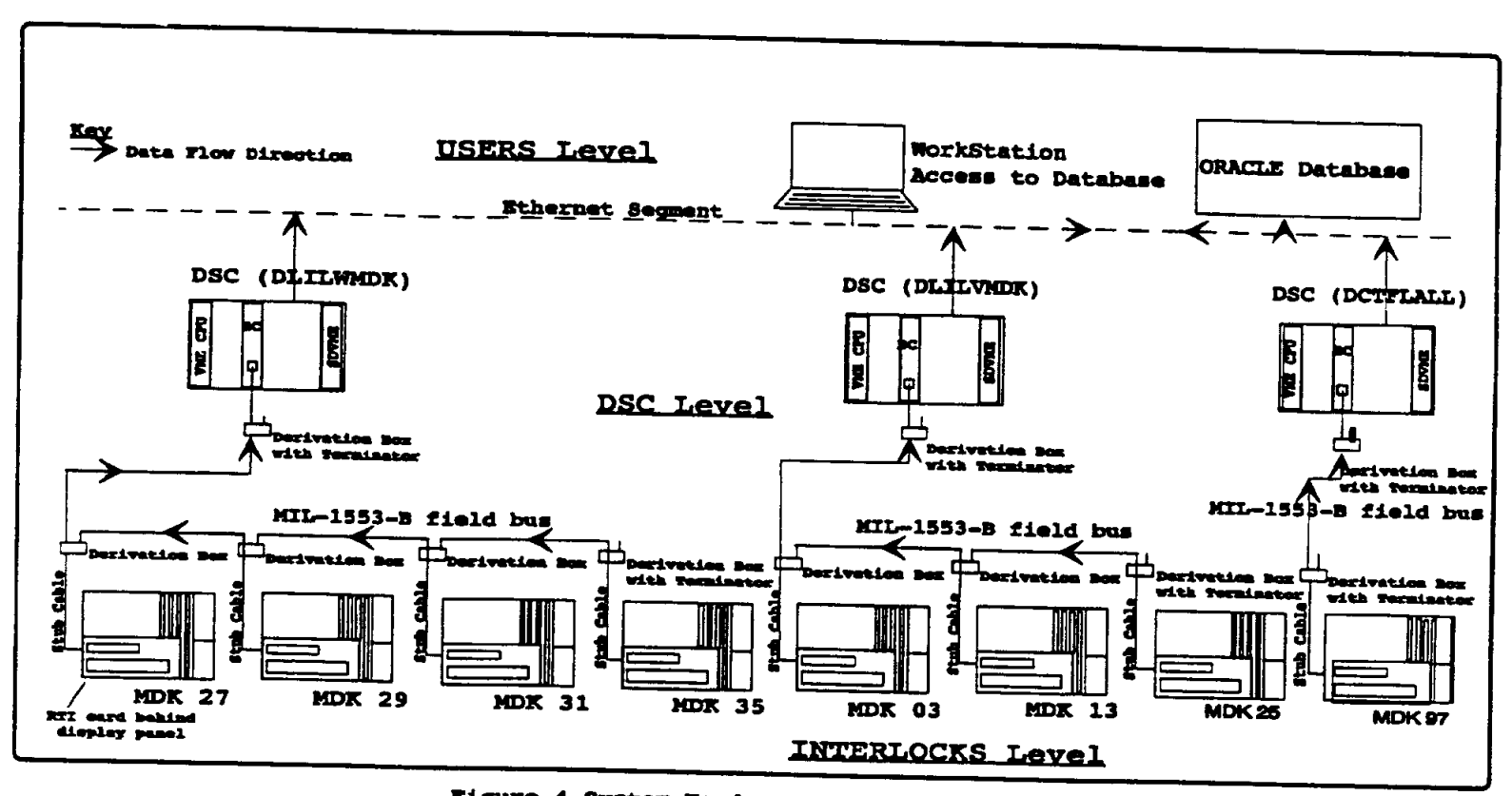

Flgure 4 Byotem Berdware Bobenetio Dilegrad

The new interlock system

The new interlock system design [2] uses three different layers of hardware and software. This basic system levels thion to be available at the three

1) Stand-alone modulator interlock protection level

2) Front-end processor (Device Stub Controller) level

3) Operational user level

The system hardware scheme based on these three levels is shown in Figure 4 .

\section{Interlock protection level}

At the interlock hardware level each G-64 interlock unit has a Remote Terminal Interface (RTI) card
plugged into its data bus. The multi-drop MIL-1553-B plugged into its data bus. The multi-drop MIL-1553-B controls system. This card up to the accelerator controls system. This enables the asynchronous exchange of data in response to comands from the Bus Controller (BC) in the front end processor (DSC). The two buffer memories on the RTI card are used as Receive and Transmit FIFo buffer stacks that hold control values, and the G-64 acquisition data. Control and status Register(CSR) bit (TB) indicates to the BC that interlock data has been loaded into the transmit buffer the transitis is only reset after the transmission is complete. A data transmission rate of 1 me MIL cable connection length used in the system.

The interlock protection micro-processor has a set of firmware assembler instructions that are used to load the faulty interlock data into the transmit buffer on the RII card. This EPROM program is triggered into action by a hardware interrupt on the occurrence of the first faulty interlock. An internal delay ensures the capture and storage of up to a maximum of subsequent cascading interlocks before the RT transmit buffer is loaded. The worst case actuisition time measured for a complete sequence acquisition interlocks was $116 \mathrm{~ms}$. However the protection response time to put the modred average non-pulsing state, after a fa the modator to a about $66 \mathrm{~ms}$ as shoum in
CH1(A): The faulty interlock.

CH2 (B): The change of output state.



Figure 5 Protection response time of modulator

Front-end processor level

At the DSC level a real-time software task has been written in $C$ language and runs under the control of the Lynx (UNIX like) operating system. The RTI buffer cards are scanned every 20 seconds by this task to check for any new data. A representation of the realtime task software algorithm is shown in Figure 6.

If new data is available from the RTI card it is taken, the CSR bit is cleared and the data reformatted and stored. The reformatting process consists removing any padding characters and dectonal points from the Ilxed length 32 byte message?

The interlock numbers are then converted into corresponding 32 bit integer values and the inIX system date and time is attached. Finalisy the UNIX to store the fault message in the Finally the position calculated and the data is stored. data table TBL is calculated and the data is stored. One table is used the specific software Equipment Module located within [4] used for LPI modulator control and acquisition. 




Figure 6 Real-time tosk algorithm representation

Operational user level

At the present moment a windows driven user program exists on the controls network [5] that has been written in Visual Basic. This enables operations and maintenance staff to see equipment faults directly maintenance staft to see equipient racular date and from a selected modulator, for a particular date and time. The displayed data is obtained either from the
current contents of the selected modulators data table TBL, or from historical data stored in the oracle database. The programs interactive display is shown in Figure 7

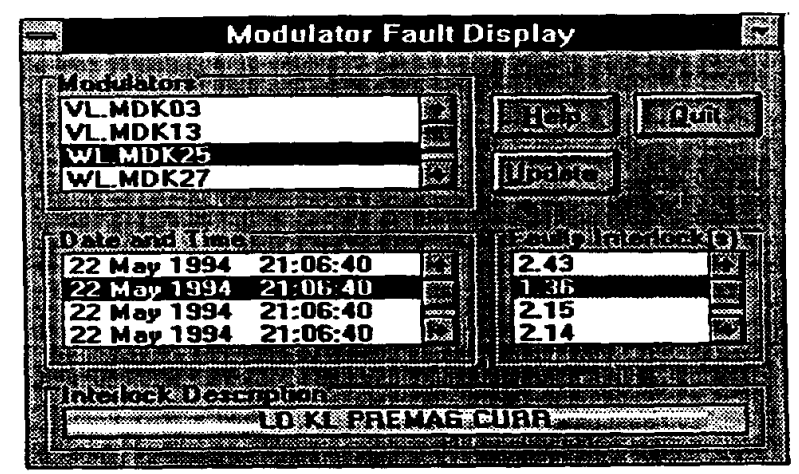

Figure 7 Fanlty interlock display

The part concerning historical fault data storage is presently being developed and uses a real-time task that must be scheduled to run daily. This collector process program will extract the fault data from the process program of each modulator, and reformat it TBI data tables of each modulator, and reformat it before sending it to the oracle database. The direct
display windows program can then access the information as required.
In addition to this a statistical analysis program is being considered that will also access the same oracle data and enable fault trends to be analysed and displayed. It is also thought that the alarm screen of the LPI process could handle usefully more detalled information on equipment faults. This data would come directly from the individual modulators data tables as failts occurred. The range of possible user sottuare to be derreloped and linked to the acquisition and storage of fault data is given in Figure 8 .

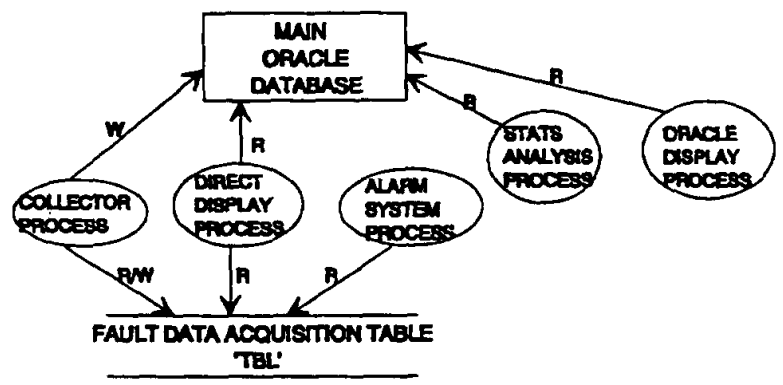

Figure 8. Range of user level coftware

Sumingy

The new interlock system has been installed in three of the CERW IPI modulator systems, since November 1993. The other six modulators will have the new interlock system installed during the course of 1994 The new equipment has performed rellably over this period with a noticeable reduction of downtime due to random faults in those modulators that have been equipped. There has also been a noticeable decrease in the fault diagnosis time since the local memories in the fault diagnosis time since the local memories of each interlo on the last eight lault seguences that have occurred. The interlock tault display program allows this information to be viewed from any PC terminal. This fault memorisation and display function allows a faster maintenance response time than in the previous relay system and improves the overall reliability and availability of the LEP process.

\section{Mckowledgement:}

The authors would like to thank G. Coudert who belped solve many problems concerning the use of the G-64 development system and EPROM programming. Thanks ar due C. Dehavay and C.H Sicard for providing help and encouragement with understanding the accelerator controls system. The new interlock equipment was carefully constructed by $H$. Meunier.

\section{References}

[1] M. Grippeling, "The design and development of a G-64 based interlock system for use with the LPI 35 M pulsed power modulators", CERN/PS/LP Note 93-01 (Tech)

[2] A. Campbel1, "Fault data Acquisition profect for the LPI modulators", CERN/PS/LP Note 93-66 (Tech)

(3) D. Francart et al, "MIL-1553-B Multidrop bus for controlling LEP and SPS equipment", CERK/SPS/ACC Note 85-26 Rev

[4] J. Cuperus, w. Heinze and C.H Sicard, "The control module handbook", CERN/PS/CO Note 91-25

[5] R. Gilmoux, "A Faulty interlock display program tor LPI modulators", CERN/PS/LP Note 94-22 (Tech) 